\title{
Characterization of a DC-driven microplasma between a capillary tube and water surface
}

Lu, Y., Xu, S. F., Zhong, X. X., Ostrikov, K., Cvelbar, U., \& Mariotti, D. (2013). Characterization of a DC-driven microplasma between a capillary tube and water surface. EPL , 102(1), 15002. http://stacks.iop.org/0295$5075 / 102 / i=1 / a=15002$

Link to publication record in Ulster University Research Portal

\section{Published in:}

EPL

Publication Status:

Published (in print/issue): 01/01/2013

\section{Document Version}

Publisher's PDF, also known as Version of record

\section{General rights}

Copyright for the publications made accessible via Ulster University's Research Portal is retained by the author(s) and / or other copyright owners and it is a condition of accessing these publications that users recognise and abide by the legal requirements associated with these rights.

\section{Take down policy}

The Research Portal is Ulster University's institutional repository that provides access to Ulster's research outputs. Every effort has been made to ensure that content in the Research Portal does not infringe any person's rights, or applicable UK laws. If you discover content in the Research Portal that you believe breaches copyright or violates any law, please contact pure-support@ulster.ac.uk. 
Characterization of a DC-driven microplasma between a capillary tube and water surface

This article has been downloaded from IOPscience. Please scroll down to see the full text article.

2013 EPL 10215002

(http://iopscience.iop.org/0295-5075/102/1/15002)

View the table of contents for this issue, or go to the journal homepage for more

Download details:

IP Address: 90.220.187.242

The article was downloaded on 16/04/2013 at 22:17

Please note that terms and conditions apply. 


\title{
Characterization of a DC-driven microplasma between a capillary tube and water surface
}

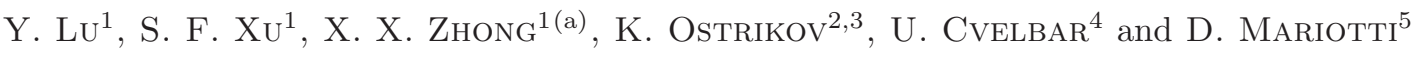 \\ ${ }^{1}$ Key Laboratory for Laser Plasmas (Ministry of Education) and Department of Physics, \\ Shanghai Jiao Tong University - Shanghai 200240, PRC \\ 2 Plasma Nanoscience Centre Australia (PNCA), CSIRO Materials Science and Engineering \\ P.O. Box 218, Lindfield, NSW 2070, Australia \\ 3 PlasmaNanoscience@ Complex Systems, School of Physics, The University of Sydney \\ Sydney, NSW 2006, Australia \\ 4 Jozef Stefan Institute - Jamova cesta 39, SI 1000 Ljubljana, Slovenia, EU \\ 5 Nanotechnology $\&$ Integrated Bio-Engineering Centre (NIBEC), University of Ulster \\ Newtownabbey, BT37 0QB, UK, EU
}

received 5 December 2012; accepted in final form 13 March 2013

published online 12 April 2013

PACS 52.50.Dg - Plasma sources

PACS 52.70.Kz - Optical (ultraviolet, visible, infrared) measurements

PACS 52.70.Ds - Electric and magnetic measurements

\begin{abstract}
A microplasma generated between a stainless-steel capillary and water surface in ambient air with flowing argon as working gas appears as a bright spot at the tube orifice and expands to form a larger footprint on the water surface, and the dimensions of the bell-shaped microplasma are all below $1 \mathrm{~mm}$. The electron density of the microplasma is estimated to be ranging from $5.32 \times 10^{9} \mathrm{~cm}^{-3}$ to $2.02 \times 10^{14} \mathrm{~cm}^{-3}$ for the different operating conditions, which is desirable for generating abundant amounts of reactive species. A computational technique is adopted to fit the experimental emission from the $\mathrm{N}_{2}$ second positive system with simulation results. It is concluded that the vibrational temperature (more than $2000 \mathrm{~K}$ ) is more than twice the gas temperature (more than $800 \mathrm{~K}$ ), which indicates the non-equilibrium state of the microplasma. Both temperatures showed dependence on the discharge parameters (i.e., gas flow and discharge current). Such a plasma device could be arranged in arrays for applications utilizing plasmainduced liquid chemistry.
\end{abstract}

Copyright (C) EPLA, 2013

Introduction. - In recent years much attention has been paid to atmospheric pressure microplasmas due to their potential application in surface processing, medical and some other applications [1-3]. Microplasmas possess characteristics such as facile, stable and efficient operation, which are highly desirable for a range of applications including nanomaterial processing. One of the major drawbacks of microplasmas is the limited plasma-surface contact area which restricts the rate of interactions; nonetheless, it may be possible to utilize array arrangements [4]. Likewise, for many applications such as those offered by plasma-induced liquid chemistry $[5,6]$, the microplasma remains a very attractive technique for scientific research in a variety of fields that include physics, chemistry, materials science and biomedicine.

(a) E-mail: xxzhong@sjtu.edu.cn
The successful application of plasmas and microplasmas in particular often rely on their non-equilibrium nature, which can offer large amounts of radical species, photons, molecular fragments, metastables, and highly energetic electrons. New chemical pathways that are not achievable with traditional plasma processes may be possible by microplasma-produced species at relatively low gas temperatures. Among all kinds of non-equilibrium plasma systems [7-10], underwater or on water discharges are interesting and promising for their value in plasma physics research [11-23] and applications in materials processing, biology, and water sterilization [5,24-26]. A range of approaches for generating plasmas in/on liquids have been explored. These include pulsed high-voltage sources, high-frequency sources and several others. It is well known that a fraction of the energy coupled to the electrons 
from the electric field goes into plasma gas heating. Because of gas flow and the high surface-to-volume ratio in microplasmas, heat can be quickly dissipated either through convection or conduction preventing the gas temperature from increasing dramatically and preserving a non-equilibrium regime [27].

On the other hand, the water electrode may be expected to contribute to the non-equilibrium state. According to Bruggeman et al., spatially resolved spectral diagnosis of a larger discharge ( $5 \mathrm{~mm}$ long) with a pin-to-water configuration [5] revealed that the gas temperature is lower near the water electrode and it was assumed that the water electrode acted as a heat sink. It is likely that heat transfer by convection is highly effective in water whereby the heat transferred through the plasma-liquid interface is quickly dissipated in the bulk of the liquid. Nonetheless, the effect of heat absorption by the water surface still needs careful consideration in the future. Although the water surface may be subject to deformation due to both the impinging flow and the applied electric field, one of the main advantages of water electrodes is that they remain chemically smooth and immune to surface degradation due to chemical reactions. In contrast, surfaces of solid electrodes are prone to surface reactions (e.g., oxidation) which can affect the stability of the discharge.

Several authors previously investigated discharge systems with electrolyte electrodes. These studies suggest that different modes, especially glow mode and multichannel mode could be ignited [11-20]. A mechanism of discharge maintenance based on the estimates of the average energy an impinging $\mathrm{H}^{+}$ion transfers to a negative ion of impurity in the electrolyte, was also proposed [11]. Recently, several other groups developed a DC-driven microplasma with a hollow cathode and water anode for nanomaterial synthesis and engineering [4,6,28,29]. However, only a few studies have been reported on the properties of this microplasma-water system. It is believed that the outcome of microplasma-assisted nanofabrication and surface engineering depends on the plasma parameters such as the electron density, electron temperature, and gas temperature. In turn, these plasma parameters are largely determined by the discharge current and the gas flow rate. These measurements are quite challenging due to the instabilities of the plasma discharges and other factors and are rarely reported; the reported values are often contradictory [27].

Here we report on the detailed optical and electrical characterization of the microplasmas for different values for the discharge current and the gas flow. Optical emission spectroscopy has been used because of its non-invasive nature [30]. The small size of microplasmas and the presence of the plasma-liquid interface make spatially resolved diagnostics quite challenging so that the averaged emission profiles are often preferred and still provide a reasonable and very useful estimate of the physical parameters.

The measured current-voltage $(I-V)$ curves suggest that the discharge is in a glow or glow-like mode. The gas

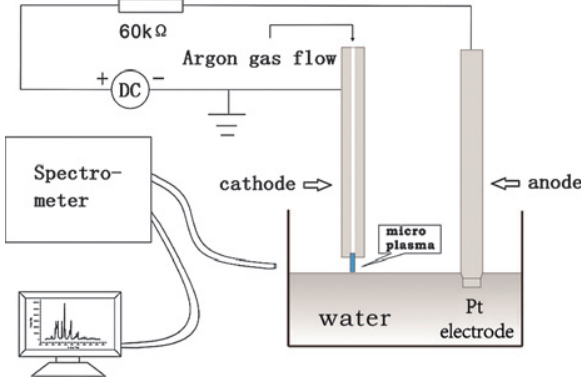

Fig. 1: (Colour on-line) Schematic diagram of the experimental set-up.

temperature of the microplasma has been determined by fitting the experimental emission spectra of the $\mathrm{N}_{2}$ second positive system with the corresponding synthetic spectra; this computational technique is valid based on the assumption that the rotational temperature equals the translational temperature. The gas temperature is found to be above $800 \mathrm{~K}$ (relatively low) and the vibrational temperature above $2000 \mathrm{~K}$, where both temperatures depend on the discharge current and on the gas flow rates; the disparity between the rotational and vibrational temperatures confirms the non-equilibrium regime. Based on the electrical current and the geometry of the microplasma the electron density is estimated to be in the rage from $5.32 \times 10^{9} \mathrm{~cm}^{-3}$ to $2.02 \times 10^{14} \mathrm{~cm}^{-3}$.

Experimental set-up. - A schematic diagram of the experimental set-up is shown in fig. 1. The circuit is driven by a high-voltage dc source (purchased from Dongwen Corporation in Tianjin, China), with a platinum electrode acting as the anode, which is immersed in water, and a stainless-steel capillary cathode with an internal diameter of 175 micrometers. A ballast resistor (about $60 \mathrm{k} \Omega$ ) limits the current and provides stability to the discharge. Argon flow through a mass flow controller is introduced into the capillary as the working gas. Gas flow rate is varied from $10 \mathrm{sccm}$ (cubic centimeters in standard conditions) to $45 \mathrm{sccm}$. The gap between the capillary and the surface of the water is adjusted to $1 \mathrm{~mm}$. The plasma is ignited when the voltage is increased to about $2000 \mathrm{~V}$. Thereafter the voltage decreases to about $600 \mathrm{~V}$ and reaches stable conditions at currents of milliamperes. Electrical characterization and emission spectroscopy were carried out and results are presented below. The AvaSpec2048FT-4-DT spectrometer was used for collecting the plasma emission.

Results and discussion. - A) Physical appearance. Photos of the microplasma at different conditions were taken with a digital camera (Canon IXUS 860 IS) and are presented in fig. 2 ; note that in the photos of fig. 2, a mirror image of the plasma can be seen reflected on the water surface, which is quite useful as it shows the plasma at the capillary orifice. Both the appearance and the $I-V$ characteristic (see next section) suggest that the microplasma 


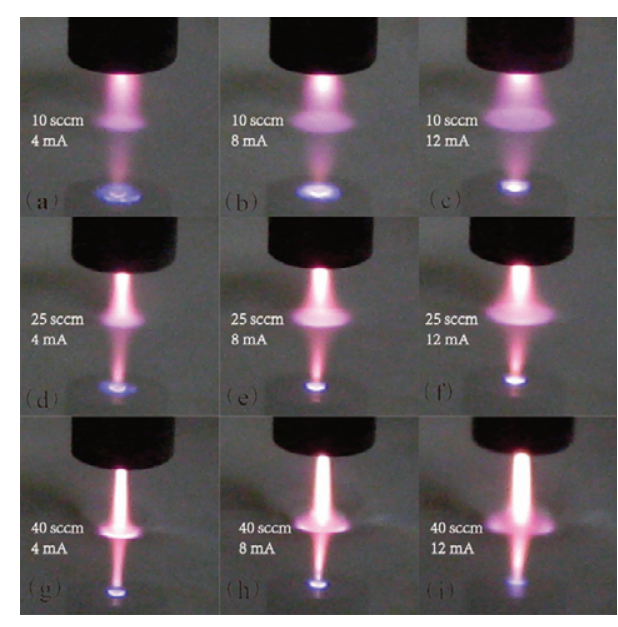

Fig. 2: (Colour on-line) Photos of the microplasmas at different gas flows and different currents: (a)-(c) $10 \mathrm{sccm}$; (d)-(f) $25 \mathrm{sccm}$; (g)-(i) $40 \mathrm{sccm}$; (a), (d), (g) $4 \mathrm{~mA}$; (b), (e), (h) $8 \mathrm{~mA}$; (c), (f), (i) $12 \mathrm{~mA}$. The gap was $1 \mathrm{~mm}$ in all cases.

is in a glow-like regime. The plasma forms a disc-shaped interface with the water surface while at the cathode a bright spot can be observed, which in some cases extends downwards to become a longer and thin plume. The inner core of the microplasma is then surrounded by a diffuse less-defined bell-shaped plasma volume which is obtained under the condition when the electric current density at the anode is constant. Moreover, from the image mirrored on the water surface, the cathode area in proximity of the capillary exit could be observed. This shows a dark space and a bright blue-coloured spots possibly within the capillary. As the current increases (see photos in fig. 2 from left to right, each row at a different flow rate), the anode spot on the water surface becomes larger with the diameter that remains below $1 \mathrm{~mm}$. When the gas flow is increased (from top to bottom in fig. 2, with each column corresponding to a different discharge current), the disc-shaped plasma-water interface appears progressively smaller and the cathode plume elongates towards the water surface. At the same time the bell-shaped diffuse discharge around the plasma inner core almost disappears at $40 \mathrm{sccm}$. It should be noted that at $40 \mathrm{sccm}$ the capillary-water gap is larger due to the deformation of the water surface caused by the gas flow pressure; this also makes it quite difficult to control the gap.

In addition, mixing of argon with ambient air and water vapour species may affect the plasma discharge. Our estimates show that the velocity of the Ar flow is $\sim 27.7 \mathrm{~m} / \mathrm{s}$ at the flow rate of $40 \mathrm{sccm}$; under such conditions the corresponding Reynolds number is $\sim 412$. These estimates suggest that the Ar jet flow is not turbulent under the conditions of our experiments. This factor reduces the potential effect of the gas mixing with the background air and water vapour. However, detailed modelling is required to elucidate the relative contributions of the gas flow and the background gas.

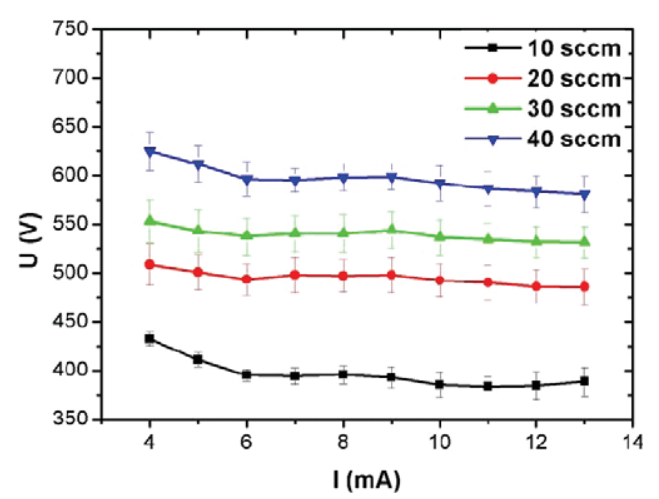

Fig. 3: (Colour on-line) Current-voltage curves of the microplasma at different gas flow rates.

Bruggeman et al. [5] reported photos of pin-to-water discharges at timescales of $100 \mathrm{~ns}$ and revealed the filamentary nature of the microplasma. Although our experimental conditions do not allow verifying this aspect, we should note that our set-up differs in important elements from the one reported by Bruggeman et al. [5], which reported on a pin-to-water configuration with $5 \mathrm{~mm}$ distance in air and with no sustained flow. In our set-up the sustained flow, the hollow cathode configuration, the use of argon and the shorter distance can all contribute to providing the conditions of a different regime. Therefore it is highly possible that our plasma does not exhibit a filamentary nature as suggested also by our $I-V$ measurements (see next section). Nonetheless, when a liquid anode is considered, the conventional relations that describe the breakdown phenomena using Paschen's law should be modified to both include the geometric and modified gas phase effects. Further experimental and theoretical studies are needed to confirm the discharge regime.

B) Electrical properties. The $I-V$ curves of the microplasma at different gas flow rates are presented in fig. 3 . The voltage and current values were initially recorded with the meters on the DC source as total voltage and total current of the entire circuit. In order to determine the voltage across the microplasma we have taken into consideration the voltage drop due to the circuit resistance including the water distributed resistance. Therefore we have measured the voltage at different current values when the capillary tube was immersed in water (table 1), i.e., we have measured the voltage and current without the plasma. For each current value, this voltage was then subtracted from the total voltage measured when the capillary was outside the water with the plasma on.

The results of the calculated voltage with the corresponding current values are presented in fig. 3 . The results of fig. 3 show that the larger is the gas flow rate the larger the plasma voltage is. One can notice that the gap between the cathode and the water surface (anode) increases by increasing the flow rate as shown in fig. 2 . Therefore, the results of fig. 3 can be explained in terms of a higher voltage required to sustain the microplasma 
Table 1: Current-voltage data when the tube is immersed in tap water.

\begin{tabular}{lc}
\hline Current $(\mathrm{mA})$ & Voltage $(\mathrm{V})$ \\
\hline 1 & 98 \\
2 & 182 \\
3 & 268 \\
4 & 353 \\
5 & 437 \\
6 & 525 \\
7 & 605 \\
8 & 690 \\
9 & 776 \\
10 & 859 \\
11 & 944 \\
12 & 1030 \\
13 & 1107 \\
14 & 1193 \\
15 & 1280 \\
16 & 1360 \\
\hline
\end{tabular}

due to the increased electron losses as the gap is increased when the flow impinging on the water surface is higher. Another source of electron losses is electron affinity to negative oxygen ions near the water.

The electron density $n_{e}$ can be estimated from electrical parameters of the discharge [10]: $n_{e}=j /\left(E \mu_{e} e\right)$, where $j$ is the current density, $E$ is the electric field, $\mu_{e}$ is the electron mobility, and $e$ is the elementary charge constant. The electron mobility $\mu_{e}$ is $4.3 \times 10^{2} \mathrm{~cm}^{2} /(\mathrm{V} \cdot \mathrm{s})$ for argon at atmospheric pressure [31]. Here we intend to estimate the lower and higher limits for the electron density and hence produce a range of possible values at the gap distance of $1 \mathrm{~mm}$ with the current in the range from $4 \mathrm{~mA}$ to $13 \mathrm{~mA}$ and the flow rate in the range from $10 \mathrm{sccm}$ to $40 \mathrm{sccm}$. The lower limit is calculated with the lowest current density (4 mA divided by the circular cross-section with $1 \mathrm{~mm}$ diameter) and the highest electrical field given by the voltage of the plasma divided by the cathode sheath at the flow rate of $40 \mathrm{sccm}$. The cathode sheath is roughly estimated to be the dark space (about $0.072 \mathrm{~mm}$ thin) as observed in the photo of the microplasma (fig. $2(\mathrm{~g})$ ). This lower limit estimate produces an electron density of $5.32 \times 10^{9} \mathrm{~cm}^{-3}$. The upper limit is calculated with the highest current density (13 mA divided by the circular cross-section with $0.175 \mathrm{~mm}$ diameter) and the lowest electrical field given by the voltage of the plasma divided by the full gap at the flow rate of $10 \mathrm{sccm}$. The upper limit is $2.02 \times 10^{14} \mathrm{~cm}^{-3}$. The electron density is therefore estimated to be between $5.32 \times 10^{9} \mathrm{~cm}^{-3}$ and $2.02 \times 10^{14} \mathrm{~cm}^{-3}$ for the different operating conditions.

Our simple model has several apparent limitations. For example, it can be improved by accounting for the anode/cathode potential drops, curvature of the impinging jet on the surface and some other factors that require
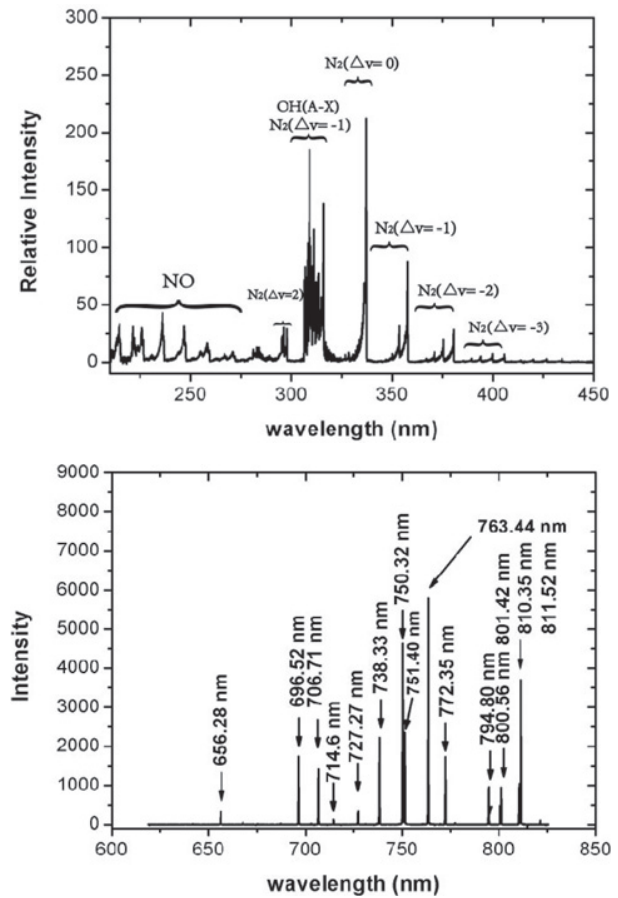

Fig. 4: Typical emission spectra of the microplasma on water with the gas flow rate at $10 \mathrm{sccm}, 5 \mathrm{~mA}$ current and a voltage of about $340 \mathrm{~V}$. In the upper panel the $\mathrm{NO}, \mathrm{OH}$ and nitrogen diatomic spectral bands are labeled. The integer number in the brackets is the change of vibrational quantum number. In the lower panel the $656.28 \mathrm{~nm}$ peak is from atomic hydrogen and the other peaks are all from argon.

a full-scale 3D modelling combined with the precise measurements of the electric-field distributions in the discharge.

C) Optical emission of the microplasma.

i) Spectral analysis. In order to examine the chemical components in the plasma, we performed optical emission spectroscopy analysis. A typical emission profile of the discharge with the current at $5 \mathrm{~mA}$ and the gas flow rate at $10 \mathrm{sccm}$ is shown in fig. 4 . From the profile of the 200-500 nm emission range, we can observe the presence of molecular NO generated bands below $273 \mathrm{~nm}$ [5], emission bands (306-310 nm) from $\mathrm{OH}(\mathrm{A}-\mathrm{X})$, and the second positive system of $\mathrm{N}_{2}(280-450 \mathrm{~nm})$; no $\mathrm{N}_{2}^{+}$band is observed. The intense $\mathrm{OH}$ bands are likely caused by the vapor phase at the plasma-water interface while the bands related to nitrogen are probably due to interaction of the plasma with the surrounding air. $\mathrm{OH}$ emission lines could also be due to air penetrating the plasma volume. However, the interface with water is expected to produce a much higher contribution to this $\mathrm{OH}$ emission. A weak signal of the $\mathrm{H}_{\alpha}$ line at $656.32 \mathrm{~nm}$ and the triplet of atomic oxygen at about $777 \mathrm{~nm}$ are also detected. Argon spectral lines in the range 400-500 $\mathrm{nm}$, i.e., the "blue lines" [10], are almost indiscernible, while Ar lines between $690 \mathrm{~nm}$ and $950 \mathrm{~nm}$, i.e., "red lines", are intense. 


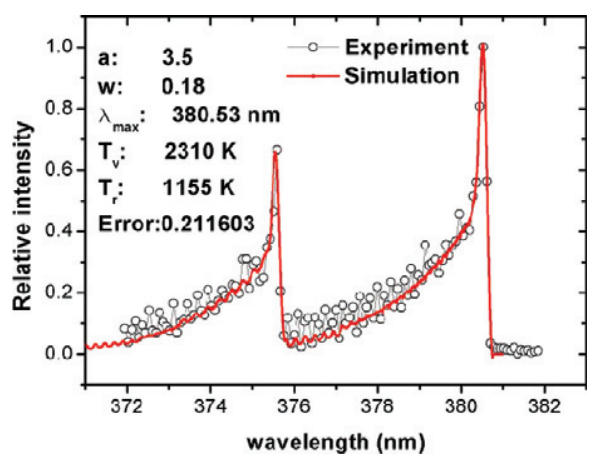

Fig. 5: (Colour on-line) Experimental and calculated spectra of two adjacent bands $\left(380 \mathrm{~nm}: \nu^{\prime}=0, \nu^{\prime \prime}=2 ; 375 \mathrm{~nm}: \nu^{\prime}=1\right.$, $\nu^{\prime \prime}=3$ ) of the $\mathrm{N}_{2}$ second positive system for a microplasma at $5 \mathrm{~mA}$ and with a gas flow rate of $10 \mathrm{sccm}$. The computational parameters are also indicated: $a$ and $w$ are used for the Phillips function [32] which determines the line shape and line width, $\lambda_{\max }$ is the wavelength of the high peak around $380 \mathrm{~nm}, T_{v}$ and $T_{r}$ the vibrational and rotational temperatures.

ii) Fitting of the spectral profile and temperature calculations. The second positive system of emission bands of nitrogen have long been utilized [32-36] to extract information from plasma spectra. The wavelength corresponding to the transition $C \nu^{\prime} J^{\prime} \rightarrow B \nu^{\prime \prime} J^{\prime \prime}$ is $[32,33]$

$$
\begin{aligned}
\lambda_{B \nu^{\prime} J^{\prime \prime}}^{C \nu^{\prime} J^{\prime}}= & \left\{n _ { a } \sum _ { p = 0 } ^ { 5 } \sum _ { q = 0 } ^ { 2 } \left\{Y_{p q}^{C}\left(\nu^{\prime}+\frac{1}{2}\right)^{p}\left[J^{\prime}\left(J^{\prime}+1\right)\right]^{q}\right.\right. \\
& \left.-Y_{p q}^{B}\left(\nu^{\prime \prime}+\frac{1}{2}\right)^{p}\left[J^{\prime \prime}\left(J^{\prime \prime}+1\right)\right]^{q}\right\}^{-1},
\end{aligned}
$$

where $\nu^{\prime}, \nu^{\prime \prime}$ are vibrational quantum numbers, $J^{\prime}, J^{\prime \prime}$ are rotational quantum numbers, $n_{a}$ is the refractive index of air. $Y_{p q}^{C}$ and $Y_{p q}^{B}$ are adopted from ref. [34]. The relative strength of the peaks in series are [33]

$$
I_{B \nu^{\prime \prime} J^{\prime \prime}}^{C \nu^{\prime} J^{\prime}}=\frac{D}{\lambda^{4}} q_{\nu^{\prime}, \nu^{\prime \prime}} \exp \left(-E_{\nu^{\prime}} / k T_{v}\right) S_{J^{\prime}, J^{\prime \prime}} \exp \left(-E_{J^{\prime}} / k T_{r}\right),
$$

where $D$ is a constant including background factors that affects the sensitivity of the spectra system, $\lambda$ is the wavelength, $k$ is Boltzmann constant, $q_{\nu^{\prime}, \nu^{\prime \prime}}$ is the FrankCondon factor, $S_{J^{\prime}, J^{\prime \prime}}$ is the Honl-London factor, $E_{\nu^{\prime}}$ and $E_{\nu^{\prime \prime}}$ are upper and lower energy states of the transition, $T_{v}$ and $T_{r}$ are the vibrational and rotational temperatures. Because of several broadening effects, e.g., instrumentrelated broadening, Stark broadening, the dense peaks in series are broadened and merge into bands as shown in fig. 4. The empirical formula of the profile of a broadened single peak is

$$
g(\Delta \lambda)=\frac{a-(2 \Delta \lambda / W)^{2}}{a+(a-2)(2 \Delta \lambda / W)^{2}},
$$

where $\Delta \lambda$ was the wavelength distance from the peak, a and $W$ are arbitrary parameters which could be adjusted to optimize the fitting [33].

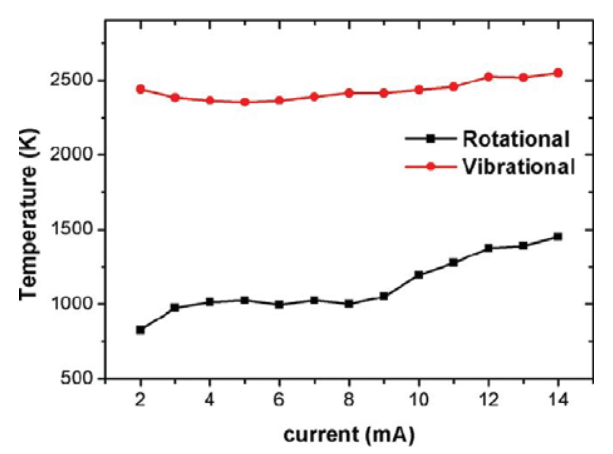

Fig. 6: (Colour on-line) Dependence of the rotational and vibrational temperatures on the discharge current. The gas flow rate is $25 \mathrm{sccm}$ and the gap is $1 \mathrm{~mm}$.

Consequently, with parameters given, a theoretical profile of the $\mathrm{N}_{2}$ bands could be synthesized. After adjusting $T_{v}, T_{r}, a, W$ and the wavelength shift due to the instrument errors to minimize the sum of squared difference between synthetic and experimental profile at every point, we obtain the rotational and vibrational temperatures as shown in fig. 5, where the synthetic spectra were compared with the experimental emission of the band at about $375 \mathrm{~nm}\left(\nu^{\prime}=1, \nu^{\prime \prime}=3\right)$ and the adjacent band at about $380 \mathrm{~nm}\left(\nu^{\prime}=0, \nu^{\prime \prime}=2\right)$ from the $\mathrm{N}_{2}$ bands of the second positive $\left(\mathrm{C}^{3} \pi_{u} \rightarrow \mathrm{B}^{3} \pi_{g}\right)$ emission system. Since the accuracy of the model used determines the accuracy of the fitting, a full discharge model is needed in the future.

Assuming that the translational temperature and rotational temperature are in equilibrium with each other, the best fit between the calculated and the experimental spectra can produce the values of gas temperature for a range of the plasma conditions. In our case, the assumption of equilibrium between the rotational and the translation temperature is justified due to the frequent collisions that take place at atmospheric pressure and due to the close gaps of ro-vibrational energy levels.

Considering that the experimental set-up collects light from several different points of the plasma volume (in particular at the plasma-air interface), it has been also assumed that the temperature gradients in the plasma are sufficiently weak. This technique has been utilized for a range of plasma studies and has been previously validated $[32-36]$.

Figure 5 presents a typical best fit between the simulated and the experimental emission profiles. In this case the rotational temperature was estimated to be $1155 \mathrm{~K}$ and the vibrational temperature $2310 \mathrm{~K}$. The vibrational temperature is more than two times the rotational temperature, which confirms that the microplasma is in a nonequilibrium state.

iii) Dependence of gas temperature and vibrational temperature on gas flow rate and current. Figures 6 and 7 report the vibrational and rotational temperatures calculated as previously described for different discharge current values and different flow rates, respectively. In 


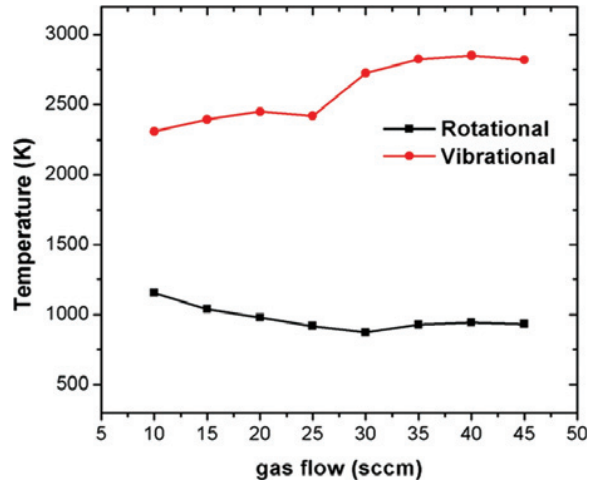

Fig. 7: (Colour on-line) Dependence of the rotational and vibrational temperatures on gas flow rate. The current is $5 \mathrm{~mA}$ and the gap is $1 \mathrm{~mm}$.

particular, in fig. 6 , as the current increases the rotational temperature increases from $800 \mathrm{~K}$ to $1400 \mathrm{~K}$, while the vibrational temperature remains relatively constant. This is probably because the vibrational temperature depends on the electron energy distribution which is closely related to the voltage, while the rotational temperature depends on the power density which is directly correlated with the current. From fig. 3 we could see that while a negative resistance is observed, the voltage changes are relatively small; this is consistent with the small variation of the vibrational temperature and its dependence on the voltage. In fig. 7, as the gas flow rate is increased from $10 \mathrm{sccm}$ to $45 \mathrm{sccm}$, the rotational temperature decreases from $1200 \mathrm{~K}$ to $900 \mathrm{~K}$, while the vibrational temperature increases from $2300 \mathrm{~K}$ to $2800 \mathrm{~K}$. These results validate that gas flow is beneficial to sustain non-equilibrium.

Conclusion. - A DC-driven microplasma with flowing argon as working gas and generated between a stainlesssteel capillary and water has been characterized. Compared to pin-to-plate configurations and other ac or pulsed high-voltage microplasmas, this microplasma has shown to be an easy-to-use configuration with very good stability and effective radicals production. The physical appearance shows a glow-like discharge characteristic and a dependence on discharge current and gas flow rates. The electron density is estimated to be in the range from $5.32 \times 10^{9} \mathrm{~cm}^{-3}$ to $2.02 \times 10^{14} \mathrm{~cm}^{-3}$ with the current in the range $4-13 \mathrm{~mA}$ and the flow rate in the range from $10 \mathrm{sccm}$ to $40 \mathrm{sccm}$ with a gap distance between the cathode and the anode of $1 \mathrm{~mm}$. The gas temperature of the plasma is estimated to be between $800 \mathrm{~K}$ and $1400 \mathrm{~K}$ depending on plasma conditions while the vibrational temperature is about twice the gas temperature; this confirms the distinct non-equilibrium state of the plasma. This microplasma device could be arranged in arrays and used for nanofabrication, nanomaterial modification [37] and other applications.
$* * *$

XXZ, YL and SFX acknowledge support from the NSFC (Grant No. 11275127, 90923005), STCSM (Grant No. 09ZR1414600) and MOST of China. DM and UC would like to acknowledge the support of the AdFutura Fellowship and ARRS. This work was partially supported by the Australian Research Council and CSIRO OCE Science Leadership Program.

\section{REFERENCES}

[1] Kong M. G., Ganguly B. N. and Hicks R. F., Plasma Sources Sci. Technol., 21 (2012) 030201.

[2] Dorai R. et al., J. Phys. D: Appl. Phys., 36 (2003) 666.

[3] Laroussi M., IEEE Trans. Plasma Sci., 37 (2009) 714.

[4] McKenna J., Patel J., Mitra S., Soin N. et al., Eur. Phys. J. Appl. Phys., 56 (2011) 24020.

[5] Bruggeman P., Liu J., Degroote J., Kong M. G. et al., J. Phys. D: Appl. Phys., 41 (2008) 215201.

[6] Richmonds C. et al., Appl. Phys. Lett., 93 (2008) 131501.

[7] Lu X. P. et al., IEEE Trans. Plasma Sci., 37 (2009) 668.

[8] Lu X. P. et al., IEEE Trans. Plasma Sci., 37 (2009) 647.

[9] Chen Q. et al., Chem. Phys. Lett., 521 (2012) 113.

[10] Tang J. et al., Phys. Plasmas, 19 (2012) 013501.

[11] Gaisin A. F. et al., High Temp., 43 (2005) 001.

[12] Gaisin A. F. et al., Plasma Phys. Rep., 37 (2011) 535.

[13] Gaisin A. F., Plasma Phys. Rep., 37 (2011) 896.

[14] Loginov N. A. et al., High Temp., 47 (2009) 603.

[15] Bagautdinova L. N. et al., High Temp., 48 (2010) 126.

[16] Gaisin Al. F. and Son E. E., High Temp., 48 (2010) 747.

[17] Gaisin A. F., High Temp., 44 (2006) 336.

[18] Gaisin A. F. et al., High Temp., 43 (2005) 810.

[19] Gaisin A. F., High Temp., 44 (2006) 792.

[20] Kayumov R. R. et al., High Temp., 46 (2008) 718.

[21] Yu L. et al., EPL, 83 (2008) 45001.

[22] Myahara T. et al., EPL, 86 (2009) 45001.

[23] Klas M. et al., EPL, 99 (2012) 57001.

[24] Kim Y. J. et al., J. Korean Phys. Soc., 60 (2012) 921.

[25] Tachibana K. et al., Plasma Sources Sci. Technol., 20 (2011) 034005.

[26] Miao S. Y. et al., IEEE Trans. Plasma Sci., 36 (2008) 136.

[27] Mariotti D. and Sankaran R. M., J. Phys. D: Appl. Phys., 43 (2010) 323001.

[28] Mariotti D. et al., Adv. Funct. Mater., 22 (2012) 954.

[29] Lee S. W. et al., J. Phys. Chem. Lett., 3 (2012) 772.

[30] Dong L. et al., Appl. Phys. Lett., 86 (2005) 161501.

[31] Raizer Y. P., Gas Discharge Physics, 2nd edition (Springer, Berlin, Germany) 1991.

[32] Phillips D. M., J. Phys. D: Appl. Phys., 9 (1976) 507.

[33] Huang X. J. et al., Phys. Plasmas, 15 (2008) 113504.

[34] Bai B. et al., J. Appl. Phys., 99 (2006) 013308.

[35] Porter R. A., J. Eletrochem. Soc., 126 (1979) 460.

[36] Tuszewski M., J. Appl. Phys., 100 (2006) 053301.

[37] Mariotti D. and Ostrikov K., J. Phys. D: Appl. Phys., 42 (2009) 092002. 